$\mathrm{e}^{+} \mathrm{e}^{-}$Collisions from Phi to Psi 2013 (PHIPSI2013)

International Journal of Modern Physics: Conference Series

Vol. 35 (2014) 1460452 (4 pages)

(C) The Authors

DOI: $10.1142 /$ S2010194514604529

\title{
ON THE SPIN CORRELATIONS OF MUONS AND TAU LEPTONS GENERATED IN THE ANNIHILATION PROCESSES $e^{+} e^{-} \rightarrow \mu^{+} \mu^{-}, e^{+} e^{-} \rightarrow \tau^{+} \tau^{-}$
}

\author{
VALERY V. LYUBOSHITZ* \\ Joint Institute for Nuclear Research \\ 141980 Dubna, Moscow Region, Russia \\ Valery.Lyuboshitz@jinr.ru \\ VLADIMIR L. LYUBOSHITZ \\ Joint Institute for Nuclear Research \\ 141980 Dubna, Moscow Region, Russia
}

Published 18 December 2014

\begin{abstract}
Using the technique of helicity amplitudes, the electromagnetic process $e^{+} e^{-} \rightarrow \mu^{+} \mu^{-}$ $\left(\tau^{+} \tau^{-}\right)$is theoretically studied in the one-photon approximation. The structure of the triplet states of the final $\left(\mu^{+} \mu^{-}\right)$system is analyzed. It is shown that in the case of unpolarized electron and positron the final muons are also unpolarized, but their spins are strongly correlated. Explicit expressions for the components of the correlation tensor of the $\left(\mu^{+} \mu^{-}\right)$system are derived. The formula for the angular correlation at the decays of final muons $\mu^{+}$and $\mu^{-}$is obtained. It is demonstrated that spin correlations of muons in the considered process have the purely quantum character, since one of the Bell-type incoherence inequalities for the correlation tensor components is always violated.
\end{abstract}

Keywords: Spin; helicity amplitudes; electron-positron pair; annihilation processes; muons; tau leptons; correlation tensor.

PACS Numbers: 13.66.De, 14.60.Ef, 14.60.Fg, 13.40.-f, 12.15.-y

\section{Helicity amplitudes for the annihilation process $e^{+} e^{-} \rightarrow \mu^{+} \mu^{-}$ and structure of the triplet states of the final $\left(\mu^{+} \mu^{-}\right)$system}

In the first non-vanishing approximation over the electromagnetic constant $e^{2} / \hbar c$, the process of conversion of the $\left(e^{+} e^{-}\right)$pair into the muon pair is described by the well-known one-photon Feynman diagram. Due to the electromagnetic current conservation, the virtual photon with a time-like momentum transfers the total

\footnotetext{
*Presenter at PHIPSI13

This is an Open Access article published by World Scientific Publishing Company. It is distributed under the terms of the Creative Commons Attribution 3.0 (CC-BY) License. Further distribution of this work is permitted, provided the original work is properly cited.
} 
angular momentum $J=1$ and negative parity. Since the internal parities of muons $\mu^{+}$and $\mu^{-}$are opposite, the $\left(\mu^{+} \mu^{-}\right)$pair is generated in the triplet states (total spin $S=1$ ) with $J=1$ and the orbital angular momenta $L=0$ and $L=2$.

The respective helicity amplitudes have the following structure:

$$
f_{\Lambda^{\prime} \Lambda}(\theta, \phi)=R_{\Lambda^{\prime} \Lambda}(E) d_{\Lambda^{\prime} \Lambda}^{(1)}(\theta) \exp (i \Lambda \phi)
$$

where $\theta, \phi$ are the polar and azimuthal angles of the flight direction of the positive muon $\left(\mu^{+}\right)$in the c.m. frame of the reaction with respect to the initial positron momentum; $d_{\Lambda^{\prime} \Lambda}^{(1)}(\theta)$ are the Wigner functions for $J=1 ; \Lambda$ is the difference of helicities of the positron and electron, coinciding with the projections of total spin and total angular momentum of the $\left(e^{+} e^{-}\right)$pair onto the direction of positron momentum in the c.m. frame; $\Lambda^{\prime}$ is the difference of helicities of the muons $\mu^{+}$and $\mu^{-}$, coinciding with the projection of total angular momentum of the $\left(\mu^{+} \mu^{-}\right)$pair onto the direction of momentum of $\mu^{+}$in the c.m. frame (see, e.g., Refs. 1,2).

In Eq. (1), $R_{\Lambda^{\prime} \Lambda}(E)=r_{\Lambda^{\prime}}^{(\mu)}(E) r_{\Lambda}^{(e)}(E)\left(\Lambda^{\prime}, \Lambda=+1,0,-1\right)$ - due to the factorizability of the Born amplitude, and here $r_{+1}^{(\mu)}=r_{-1}^{(\mu)}=r_{1}^{(\mu)}, r_{+1}^{(e)}=r_{-1}^{(e)}=r_{1}^{(e)}$ owing to the space parity conservation in the electromagnetic interactions . Further, in accordance with the structure of electromagnetic current for the pairs $\left(e^{+} e^{-}\right)$ and $\left(\mu^{+} \mu^{-}\right)$in the c.m. frame, ${ }^{1}$ the following relations are valid:

$$
r_{0}^{(\mu)}=\frac{m_{\mu}}{E} r_{1}^{(\mu)}=\sqrt{1-\beta_{\mu}^{2}} r_{1}^{(\mu)}, \quad r_{0}^{(e)}=\frac{m_{e}}{E} r_{1}^{(e)},
$$

where $m_{\mu}, m_{e}$ are the muon and electron masses and $\beta_{\mu}$ is the muon velocity in the c.m. frame. Thus, since we always have $E \geq m_{\mu} \gg m_{e}$ for the given process, the contribution of $\left(e^{+} e^{-}\right)$-states with equal helicities can be neglected, i.e. $R_{\Lambda 0}(E) \approx 0$.

The one-photon diagram calculation gives (here $e$ is the electron charge) :

$$
r_{1}^{(\mu)}(E)=r_{1}^{(e)}(E)=\frac{|e|}{\sqrt{2 E}} .
$$

Using the expressions (1)-(3) and the formulas for $d$-functions at $J=1^{1,2}$, we may find the effective cross section of the reaction $e^{+} e^{-} \rightarrow \mu^{+} \mu^{-}$and the angular distribution of muon emission, normalized by unity, in the c.m. frame (see Ref. 3) .

Taking into account Eqs. (1)-(3), it is clear that, in the cases of total polarization of both the positron and electron along the positron momentum in the c.m. frame and in the direction being antiparallel to the positron momentum, the $\left(\mu^{+} \mu^{-}\right)$ system is produced, respectively, in the triplet states of the following form: ${ }^{3}$

$$
\begin{aligned}
& |\Psi\rangle^{(+1)}=\frac{\sqrt{2}}{\sqrt{2-\beta_{\mu}^{2} \sin ^{2} \theta}}\left(\frac{1+\cos \theta}{2}|+1\rangle-\sqrt{1-\beta_{\mu}^{2}} \frac{\sin \theta}{\sqrt{2}}|0\rangle+\frac{1-\cos \theta}{2}|-1\rangle\right) . \\
& |\Psi\rangle^{(-1)}=\frac{\sqrt{2}}{\sqrt{2-\beta_{\mu}^{2} \sin ^{2} \theta}}\left(\frac{1-\cos \theta}{2}|+1\rangle+\sqrt{1-\beta_{\mu}^{2}} \frac{\sin \theta}{\sqrt{2}}|0\rangle+\frac{1+\cos \theta}{2}|-1\rangle\right) .
\end{aligned}
$$


Here $\beta_{\mu}$ is the velocity of each of the muons - just as in (2), and $|+1\rangle,|-1\rangle,|0\rangle$ are the states with the projection of total spin of the $\left(\mu^{+} \mu^{-}\right)$pair onto the direction of momentum of $\mu^{+}$in the c.m. frame, equaling $+1,-1$ and 0 , respectively.

\section{Correlation tensor of the $\left(\mu^{+} \mu^{-}\right)$pair and violation of "classical" incoherence inequalities}

If the positron and electron are not polarized, then, since $r_{0}^{(e)} \approx 0$, the final state of the $\left(\mu^{+} \mu^{-}\right)$pair represents an incoherent mixture of spin states $|\Psi\rangle^{(+1)}$ and $|\Psi\rangle^{(-1)}$, each of them being realized with the relative probability of $1 / 2$.

The components of the correlation tensor for two particles with spin $1 / 2$ are defined as: $T_{i k}=\left\langle\hat{\sigma}_{i}^{(1)} \otimes \hat{\sigma}_{k}^{(2)}\right\rangle(i, k \rightarrow\{1,2,3\} \rightarrow\{x, y, z\}$; the symbol $\langle\ldots\rangle$ denotes the averaging over the quantum ensemble). For the considered final $\left(\mu^{+} \mu^{-}\right)$pair, the axis $z$ is directed along the momentum of $\mu^{+}$in the c.m. frame of the reaction $e^{+} e^{-} \rightarrow \mu^{+} \mu^{-}$, and the axis $y$ - along the normal to the reaction plane.

It is easy to see that, in the case of unpolarized primary positron and electron, the produced muons $\mu^{+}$and $\mu^{-}$are also unpolarized but their spins are correlated, and the correlation tensor components have the following form ${ }^{3}$ (see also Ref. 5):

$$
\begin{gathered}
T_{x x}^{\left(\mu^{+} \mu^{-}\right)}=\frac{\left(2-\beta_{\mu}^{2}\right) \sin ^{2} \theta}{2-\beta_{\mu}^{2} \sin ^{2} \theta}, \quad T_{z z}^{\left(\mu^{+} \mu^{-}\right)}=\frac{2 \cos ^{2} \theta+\beta_{\mu}^{2} \sin ^{2} \theta}{2-\beta_{\mu}^{2} \sin ^{2} \theta}, \\
T_{y y}^{\left(\mu^{+} \mu^{-}\right)}=-\frac{\beta_{\mu}^{2} \sin ^{2} \theta}{2-\beta_{\mu}^{2} \sin ^{2} \theta}, \quad T_{x z}^{\left(\mu^{+} \mu^{-}\right)}=-\frac{\left(1-\beta_{\mu}^{2}\right)^{1 / 2} \sin 2 \theta}{2-\beta_{\mu}^{2} \sin ^{2} \theta}, \quad T_{x y}=T_{y z}=0 .
\end{gathered}
$$

Just as it should hold for triplet states, the "trace" of the correlation tensor is: $T^{\left(\mu^{+} \mu^{-}\right)}=T_{x x}^{\left(\mu^{+} \mu^{-}\right)}+T_{y y}^{\left(\mu^{+} \mu^{-}\right)}+T_{z z}^{\left(\mu^{+} \mu^{-}\right)}=1$. Let us note that the "trace" of the correlation tensor $T$ determines the angular correlation between flight directions for the products of decay of two unstable particles with spin $1 / 2$ under the space parity nonconservation (see Ref. 4-8). In particular, for the decays of the muons $\mu^{-}$ and $\mu^{+}\left(\mu^{-} \rightarrow e^{-} \nu_{\mu} \overline{\nu_{e}}, \mu^{+} \rightarrow e^{+} \overline{\nu_{\mu}} \nu_{e}\right)$, produced in the process $e^{+} e^{-} \rightarrow \mu^{+} \mu^{-}$, we obtain $^{3}$ (in accordance with the general formula for angular correlation ${ }^{4-8}$ ):

$$
d W^{\left(\mu^{+} \mu^{-}\right)}=\frac{1}{2}\left(1+\frac{\alpha_{1} \alpha_{2} T}{3} \cos \delta\right) d(-\cos \delta)=\frac{1}{2}\left(1-\frac{1}{27} \cos \delta\right) d(-\cos \delta) .
$$

Here $\alpha_{1}$ and $\alpha_{2}$ are the coefficients of angular asymmetry for the decays of the first $\left(\mu^{-}\right)$and second $\left(\mu^{+}\right)$particle (here we have $\alpha_{1}=-1 / 3, \alpha_{2}=+1 / 3^{9}$ ), $\cos \delta=\mathbf{n}_{1} \mathbf{n}_{2}, \mathbf{n}_{1}$ and $\mathbf{n}_{2}$ are unit vectors along the momenta of particles formed in the first and second decay (in our case $-e^{-}$and $e^{+}$), which are defined, respectively, in the rest frames of the first and second unstable particle and specified with respect to a unified system of spatial coordinate axes ${ }^{7,8}$ (for further details, see also Ref. 3 ).

Regarding the components (6) of the correlation tensor, we observe here the violation of the "classical" incoherence inequalities (for incoherent mixtures of factorizable states of two spin-1/2 particles, the modulus of sum of any two (and three) 
diagonal components of the correlation tensor cannot exceed unity $\left.{ }^{4,5}\right)$. Indeed, according to (6), one of the incoherence inequalities is always violated at $\theta \neq 0:^{3}$

$$
T_{x x}^{\left(\mu^{+} \mu^{-}\right)}+T_{z z}^{\left(\mu^{+} \mu^{-}\right)}=1-T_{y y}^{\left(\mu^{+} \mu^{-}\right)}=\frac{2}{2-\beta_{\mu}^{2} \sin ^{2} \theta}>1 .
$$

Thus, we see that the spin correlations of muons in the process $e^{+} e^{-} \rightarrow \mu^{+} \mu^{-}$ have the strongly pronounced quantum character.

Certainly, all the above consideration can be wholly applied also to the process $e^{+} e^{-} \rightarrow \tau^{+} \tau^{-}-$with the replacements $m_{\mu} \rightarrow m_{\tau}, \beta_{\mu} \rightarrow \beta_{\tau}$.

Finally, it should be noted that at very high energies the annihilation processes $e^{+} e^{-} \rightarrow \mu^{+} \mu^{-}, \tau^{+} \tau^{-}$are conditioned not only by the electromagnetic interaction through the virtual photon, but also by the weak interaction of neutral currents through the virtual $Z^{0}$ boson. ${ }^{9}$ The interference of amplitudes of the purely electromagnetic and weak interaction leads to the charge asymmetry in lepton emission and to the effects of space parity violation. Due to the contribution of weak interaction, in the case of unpolarized electron and positron the final leptons acquire the longitudinal polarization; the polarization vectors of the positively and negatively charged leptons are the same, and their average helicities have different signs on account of the opposite directions of momenta in the c.m. frame (see Ref. 3) .

The structure of the correlation tensor of the final leptons is, on the whole, similar to that for the case of purely electromagnetic annihilation at very high energies - see Eqs. (6) at $\beta_{\mu} \rightarrow 1\left(\beta_{\tau} \rightarrow 1\right)$. In doing so, the nonzero components of the correlation tensor are: $T_{z z}=1, T_{x x}=-T_{y y}$, as before (but, of course, the expression for $T_{x x}$ changes $\left.^{3}\right)$. Again, one of the incoherence inequalities for the correlation tensor components is violated: $T_{x x}+T_{z z}>1 .^{3}$

\section{References}

1. V. B. Berestetsky, E. M. Lifshitz and L. P. Pitaevsky, Kvantovaya Electrodinamika (Quantum Electrodynamics), in Russian (Nauka, Moscow, 1989), §§68, 69, 81.

2. A. M. Baldin, V. I. Gol'dansky, V. M. Maksimenko and I. L. Rosental', Kinematika Yadernykh Reaktsij (Kinematics of Nuclear Reactions), in Russian (Atomizdat, Moscow, 1968), part II, $\S 11$.

3. V. L. Lyuboshitz and V. V. Lyuboshitz, Yad. Fiz. 72, 340 (2009) [ Phys. At. Nucl. 72, 311 (2009) ].

4. V. L. Lyuboshitz, in Proc. XXXIV Winter School of PNPI "Physics of Atomic Nuclei and Elementary Particles" (St. Petersburg, 2000), p. 402.

5. R. Lednicky and V. L. Lyuboshitz, Phys. Lett. B 508, 146 (2001).

6. G. Alexander and H. J. Lipkin, Phys. Lett. B 352, 162 (1995).

7. R. Lednicky, V. V. Lyuboshitz and V. L. Lyuboshitz, Yad. Fiz. 66, 1007 (2003) [ Phys. At. Nucl. 66, 975 (2003) ].

8. R. Lednicky, V. L. Lyuboshitz and V. V. Lyuboshitz, Czech. J. Phys. (Suppl. B) 54, p. B43 (2004).

9. L. B. Okun, Leptony i kwarki (Leptons and Quarks), in Russian (Nauka, Moscow, 1990), $\S \S 3,8,22$. 